\title{
REPRESENTATION OF PSEUDOANALYTIC FUNCTIONS IN THE SPACE
}

\author{
P. BERGLEZ \\ Department of Mathematics, \\ Graz University of Technology, \\ Graz, Austria \\ E-mail: berglez@tugraz.at
}

\begin{abstract}
We consider a generalized Vekua equation in biquaternionic formalism where the CauchyRiemann operator is replaced by the differential operator $D$ of Dirac. For particular classes we construct differential operators of higher order which give a relation between the monogenic functions as solutions of $D w=0$ and the generalized pseudoanalytic functions as solutions of the generalized Vekua equation. This is done by considering a corresponding differential equation of second order. Using generating functions in the sense of L. Bers we can give further representations of such functions and we can obtain related pseudoanalytic functions of the second kind as solutions of another differential equation of first order.
\end{abstract}

\section{Introduction}

We denote by $\mathbb{H}(\mathbb{C})$ the algebra of complex quaternions (so called biquaternions) defined by

$$
\mathbb{H}(\mathbb{C})=\left\{a \mid a=a_{0}+a_{1} i_{1}+a_{2} i_{2}+a_{3} i_{3}\right\}
$$

where the $i_{k}$ are the standard basic quaternions with $i_{3}=i_{1} i_{2}, a_{k} \in \mathbb{C}$, and the complex imaginary unit $i$ commutes with the $i_{k}, k=1,2,3$. The quaternionic conjugation is given by $\bar{a}:=a_{0}-a_{1} i_{1}-a_{2} i_{2}-a_{3} i_{3}$. Let $\mathcal{S}$ denote the subset of zero divisors from $\mathbb{H}(\mathbb{C})$.

Generalizing the Cauchy-Riemann operator $\partial_{\bar{z}}=\frac{1}{2}\left(\partial_{x}+i \partial_{y}\right)$ we introduce the Dirac operator

$$
D:=\sum_{k=1}^{3} i_{k} \partial_{k} \quad \text { where } \quad \partial_{k}=\frac{\partial}{\partial x_{k}}
$$

(see e.g. K. Gürlebeck and W. Sprössig ${ }^{2}$ ). For functions $f: \mathbb{R}^{3} \rightarrow \mathbb{H}(\mathbb{C})$ the set $\operatorname{ker} D$ defines the class of regular functions with respect to $D$. Since the algebra of quaternions is non-commutative we have to distinguish between $f \in \operatorname{Ker} D$ such that $D f=0$ or $0=f D$. Such functions are called left monogenic or right monogenic respectively. 
H.R. Malonek ${ }^{5,6}$ extended the concept of the so called $(F, G)$-derivative in the sense of L. Bers to quaternionic-valued functions to study a spatial version of pseudoanalytic functions. He proved that these pseudoanalytic functions in the space obey a generalized Vekua equation.

The generalized pseudoanalytic functions considered here are solutions of the generalized Vekua equation

$$
D^{*} w=\frac{m}{x_{1}} \bar{w}, m \in \mathbb{N}
$$

with $D^{*}=-i_{1} D$ for which we give a general representation theorem using differential operators. Thus we establish a relation between the monogenic functions and the pseudoanalytic functions in the space. Furthermore relations between the solutions of such differential equations with different parameters $m$ are proved.

Finally we give generating pairs for such classes of generalized pseudoanalytic functions and we represent generalized pseudoanalytic functions of the second kind also.

For example such pseudoanalytic functions in the space are of interest in treating the Dirac equation with a vectorial electromagnetic potential (see e.g. V.V. Kravchenko et al. $^{4}$ ) or the multidimensional stationary Schrödinger equation (see V.V. Kravchenko ${ }^{3}$ ).

\section{Differential operators for the solutions}

We first consider the generalized Vekua equation

$$
D^{*} w=\varphi\left(x_{1}\right) \bar{w}
$$

and look for a solution of the form

$$
w=\sum_{k=0}^{m} A_{k}\left(x_{1}\right)\left(g{\overline{D^{*}}}^{k}\right)+\sum_{k=0}^{n} B_{k}\left(x_{1}\right)\left(D^{* k} \bar{g}\right)
$$

with $m, n \in \mathbb{N}$ where $D g=0$ i.e. the function $g$ is assumed to be left monogenic and $\overline{D^{*}}=\partial_{x_{1}}+i_{3} \partial_{x_{2}}-i_{2} \partial_{x_{3}}$ is used. Doing so we are led to the relation

$$
n=m-1 \text {, }
$$

and the following conditions

$$
\begin{aligned}
A_{m}^{\prime}\left(x_{1}\right) & =0 \\
A_{k}^{\prime}\left(x_{1}\right) & =\varphi\left(x_{1}\right) B_{k}\left(x_{1}\right), k=m-1, \ldots, 0, \\
B_{m-1}\left(x_{1}\right) & =\varphi\left(x_{1}\right) A_{m}\left(x_{1}\right), \\
B_{k-1}\left(x_{1}\right) & =-B_{k}^{\prime}\left(x_{1}\right)+\varphi\left(x_{1}\right) A_{k}\left(x_{1}\right), k=m-1, \ldots, 1, \\
0 & =-B_{0}^{\prime}\left(x_{1}\right)+\varphi\left(x_{1}\right) A_{0}\left(x_{1}\right) .
\end{aligned}
$$

The conditions (3) form a system of $2 m+2$ ordinary differential equations for the $2 m+1$ functions $A_{k}, k=0, \ldots, m, B_{k}, k=0, \ldots, m-1$, which is overdetermined in 
general. This means that in the general case it is not possible to obtain solutions of this generalized Vekua equation in the form (2). But as we will see in the following there are factors $\varphi\left(x_{1}\right)$ in (1) such that the system (3) can be satisfied. We can assume that there will be only a few classes of differential equations of such type for which a representation of the solutions using differential operators acting on monogenic functions exists. Indeed it is a disadvantage of the method presented here that the differential equations which can be solved by this method are very particular. On the other hand we have the advantage that the application of such differential operators is a method consisting of a finite number of processes which can be carried out for a wide range of monogenic functions in an explicite and easy way.

For further investigations we will not consider the system (3) but we will turn over to a differential equation of second order to obtain a general representation theorem for the solutions of such a generalized Vekua equation.

\section{A differential equation of second order}

Consider the generalized Vekua equation

$$
D^{*} w=\frac{m}{x_{1}} \bar{w}, m \in \mathbb{N} .
$$

We apply the operator $\overline{D^{*}}$ from the right to this equation and get the differential equation of second order

$$
D^{*} w \overline{D^{*}}+\frac{1}{x_{1}} D^{*} w-\frac{m^{2}}{x_{1}^{2}} w=0 .
$$

For this differential equation we will deduce the general solution now. First let us consider the differential equation

$$
D^{*} w \overline{D^{*}}-\frac{1}{x_{1}} D^{*} w-\frac{m^{2}-1}{x_{1}^{2}} w=0
$$

which is associated to Eq. (5) in the following manner. We denote by $\Omega$ a suitable domain in $\mathbb{R}^{3}$ not containing the plane $x_{1}=0$ and by $\mathcal{L}_{m}(\Omega)$ and $\mathcal{L}_{m-1}(\Omega)$ the set of solutions of the differential equations (5) and (6) respectively defined in $\Omega$. For $w \in \mathcal{L}_{m}(\Omega)$ we have

$$
u=R w:=\frac{x_{1}^{2}}{m^{2}} D^{*} w \in \mathcal{L}_{m-1}(\Omega)
$$

and for $w \in \mathcal{L}_{m-1}(\Omega)$ we have

$$
v=S w:=w \overline{D^{*}}-\frac{1}{x_{1}} w \in \mathcal{L}_{m}(\Omega)
$$

and furthermore

$$
S \circ R=\operatorname{id}_{\mathcal{L}_{m}(\Omega)}, \quad R \circ S=\operatorname{id}_{\mathcal{L}_{m-1}(\Omega)}
$$

holds. Thus we have the following 
Lemma 3.1. The mapping

$$
R: \mathcal{L}_{m}(\Omega) \rightarrow \mathcal{L}_{m-1}(\Omega)
$$

with $R$ from (7) is an isomorphism. Its inverse $R^{-1}$ is given by

$$
R^{-1}=S \text {. }
$$

Now let $\mathcal{L}_{m-k}(\Omega)$ denote the set of solutions of the differential equation

$$
D^{*} w_{m-k} \overline{D^{*}}+\frac{1-2 k}{x_{1}} D^{*} w_{m-k}-\frac{m^{2}-k^{2}}{x_{1}^{2}} w_{m-k}=0, k=0,1,2, \ldots
$$

With $k=0$ we get Eq. (5). By iterated application of the mapping $R_{m-k}$ : $\mathcal{L}_{m-k}(\Omega) \rightarrow \mathcal{L}_{m-k-1}(\Omega)$ with $R_{m-k} u:=\frac{x_{1}^{2}}{m^{2}-k^{2}} D^{*} u$, which in general is an isomorphism too, the solutions of these differential equations are related.

With $k=m$ in (9) we get the equation

$$
D^{*} w_{0} \overline{D^{*}}+\frac{1-2 m}{x_{1}} D^{*} w_{0}=0 .
$$

Let the operator $I$ generate the antiderivative from a biquaternionic-valued function that means

$$
I(f)=F \quad \text { with } \quad D^{*} F=f .
$$

With this we have

$$
w_{0}=I\left(x_{1}^{2 m-1} \hat{h}\right)+g
$$

where the functions $\hat{h}$ and $g$ obey the conditions $\hat{h} \overline{D^{*}}=0$ and $D^{*} g=0$ respectively. By

$$
w \equiv w_{m}=S_{m-1} \circ \ldots \circ S_{0} w_{0}
$$

with $S_{k}: \mathcal{L}_{k-1}(\Omega) \rightarrow \mathcal{L}_{k}(\Omega), S_{k} u:=u \overline{D^{*}}-\frac{2 m-2 k-1}{x_{1}} u$ we get the solutions of Eq. (5). With $w_{0}$ from (10) and the substitution $\hat{h}=D^{* 2 m} h$, where $h \overline{D^{*}}=0$ holds, we can transform the representation (11) for $w$ into the form

$$
w=\sum_{j=0}^{m} \frac{(-1)^{m-j}(2 m-1-j) !}{j !(m-j) ! x_{1}^{m-j}}\left(g{\overline{D^{*}}}^{*}\right)+\sum_{j=0}^{m-1} \frac{(-1)^{m-1-j}(2 m-1-j) !}{j !(m-1-j) ! x_{1}^{m-j}}\left(D^{* j} h\right) .
$$

Now we have the following

\section{Theorem 3.1.}

(1) For each solution of (5) there exist two functions $g$ left monogenic in $\Omega$ (i.e. $D^{*} g=0$ ) and $h$ right antimonogenic in $\Omega$ (i.e. $h \overline{D^{*}}=0$ ) respectively such that the representation (12) holds.

(2) For each function $g$ left monogenic in $\Omega$ and $h$ right antimonogenic in $\Omega$ the expression in (12) represents a solution of (5) in $\Omega$. 
(3) For a solution $w$ of (5) in the form (12) the functions

$$
\left(g{\overline{D^{*}}}^{2 m}\right)=\frac{m}{x_{1}^{2 m+1}}\left(P^{m}\left(x_{1} w\right)\right) \quad \text { with } \quad P w=x_{1}^{2}\left(w \overline{D^{*}}\right)
$$

and

$$
\left(D^{* 2 m} h\right)=\frac{1}{m x_{1}^{2 m+1}}\left(Q^{m+1} w\right) \quad \text { with } \quad Q w=x_{1}^{2}\left(D^{*} w\right)
$$

are determined uniquely.

Between the solutions of Eq. (5) with different parameters $m$ there exist interesting relations. Let $\mathcal{F}_{m}(\Omega)$ be the set of solutions of (5)

$$
D^{*} w \overline{D^{*}}+\frac{1}{x_{1}} D^{*} w-\frac{m}{x_{1}^{2}} w=0
$$

and $\mathcal{F}_{m \pm 1}(\Omega)$ the set of solutions of the differential equation

$$
D^{*} w \overline{D^{*}}+\frac{1}{x_{1}} D^{*} w-\frac{(m \pm 1)^{2}}{x_{1}^{2}} w=0
$$

For $w \in \mathcal{F}_{m}(\Omega)$ we have

$$
\begin{aligned}
& v_{1}=w \overline{D^{*}}-D^{*} w \in \mathcal{F}_{m}(\Omega), \\
& v_{2}=w \overline{D^{*}}+\frac{m-1}{m} D^{*} w+\frac{2 m-1}{x_{1}} w \in \mathcal{F}_{m-1}(\Omega), \\
& v_{3}=w \overline{D^{*}}+\frac{m+1}{m} D^{*} w-\frac{2 m+1}{x_{1}} w \in \mathcal{F}_{m+1}(\Omega) .
\end{aligned}
$$

\section{Representation of pseudoanalytic functions in the space}

Now we can deduce a representation for the solutions of Eq. (4). From the preceeding section we see that among the solutions of (5) we find the solutions of the generalized Vekua equation (4). Inserting the solution $w$ of (5) according to (12) into Eq. (4) we are led to the condition

$$
g-m \bar{h}=0
$$

After the substitution $g=m f, f$ left monogenic, we have the following representation for the solutions of Eq. (4)

$$
w=\sum_{j=0}^{m} \frac{(-1)^{m-j}(2 m-1-j) !}{j !(m-j) ! x_{1}^{m-j}}\left[m\left(f{\overline{D^{*}}}^{j}\right)-(m-j)\left(D^{* j} \bar{f}\right)\right]
$$

and we can prove the following 


\section{Theorem 4.1.}

(1) For each function $f$ left monogenic in $\Omega \subset \mathbb{R}$ (i.e. Df $=0$ ) the function $w$ according to (13) is a solution of the generalized Vekua equation (4)

$$
D^{*} w=\frac{m}{x_{1}} \bar{w}, m \in \mathbb{N} .
$$

(2) For each solution $w$ of equation (4) defined in $\Omega$ there exists a function $f$ monogenic in $\Omega$ such that (13) holds.

(3) The function $f$ in (13) is not determined uniquely by $w$. Only the expression $\left(f{\overline{D^{*}}}^{2 m}\right)$ is determined uniquely by

$$
\left(f{\overline{D^{*}}}^{2 m}\right)=\frac{1}{x_{1}^{m}}\left[\left(x_{1}^{m} w\right){\overline{D^{*}}}^{m}\right] .
$$

There exist connections between the solutions of the generalized Vekua equation (4) with different parameters. Let

$$
\mathcal{V}_{m}(\Omega)=\left\{w: D^{*} w=\frac{m}{x_{1}} \bar{w}\right\}
$$

be se set of the solutions of Eq. (4) defined in $\Omega$. Then we have

$$
\begin{aligned}
& u_{1}=w \overline{D^{*}}+\frac{m+1}{x_{1}} \bar{w}-\frac{2 m+1}{x_{1}} w \in \mathcal{V}_{m+1}(\Omega), \\
& u_{2}=w \overline{D^{*}}+\frac{m-1}{x_{1}} \bar{w}+\frac{2 m-1}{x_{1}} w \in \mathcal{V}_{m-1}(\Omega), \\
& u_{3}=w \overline{D^{*}}-\frac{m}{x_{1}} \bar{w} \in \mathcal{V}_{-m}(\Omega) .
\end{aligned}
$$

With the last relation solutions may be obtained for Eq. (4) even in the case when $m$ is a negative integer.

\section{Generating pairs}

H.R. Malonek ${ }^{6}$ extended the concept of generating functions introduced by L. Bers ${ }^{1}$ for the representation of pseudoanalytic functions in the space. Let $\mathbb{H}_{k}$ denote the set of reduced complex quaternions which have the form $a=a_{0}+a_{k} i_{k}$, $a_{0}, a_{k} \in \mathbb{C}, k=1,2$. Two pairs of functions $\mathcal{H}_{1}=(F, G), \mathcal{H}_{2}=(M, N)$ with $F, G \in \mathbb{H}_{1}, M, N \in \mathbb{H}_{2}, F, G, M, N \notin \mathcal{S}$, are called generating pairs for the solutions of (4) if $G \bar{F}-\bar{G} F \neq 0, N \bar{M}-\bar{N} M \neq 0$ in $\Omega$ and the products $F G, F N, G M, G N$ are solutions of the generalized Vekua equation (4). For $m=1$ such generating pairs are given by

$$
\begin{array}{ll}
F=1 & M=x_{1}^{-m}\left(x_{1}-(2 m-1) x_{3} i_{2}\right), \\
G=i_{1} & N=x_{1}^{-m} i_{2} .
\end{array}
$$

With these functions the solutions of Eq. (4) can be represented in the form

$$
w=F(M \phi+N \psi)+G(M \mu+N \nu)
$$


where $\phi, \psi, \mu, \nu$ are suitable complex valued functions, which are determined uniquely by the solution $w$.

\section{Pseudoanalytic functions of the second kind in the space}

Generalizing the concept of L. Bers the corresponding spatial version of a pseudoanalytic function of second kind $\omega$ is defined as

$$
\omega(x)=\phi(x)+\mu(x) i_{1}+\psi(x) i_{2}+\nu(x) i_{3}
$$

(cf. H.R. Malonek ${ }^{5}$ ). In the case of our particular pairs of generating functions (again for $m=1$ ) it obeys the differential equation

$$
\left(D^{*} \omega\right)\left(x_{1}+1-(2 m-1) x_{3} i_{2}\right)+J_{2}\left(\overline{D^{*}} \omega\right)\left(x_{1}-1-(2 m-1) x_{3} i_{2}\right)=0
$$

where $J_{2}$ denotes the linear mapping $J_{2}: \mathbb{H}(\mathbb{C}) \rightarrow \mathbb{H}(\mathbb{C})$ with $J_{2}\left(i_{1}\right)=i_{1}$ and $J_{2}\left(i_{2}\right)=-i_{2}$.

A solution can be obtained by

$$
\omega=x_{1}^{m-1}\left(w_{0}+i_{1} w_{1}+i_{2}\left(x_{1} w_{2}+(2 m-1) x_{3} w_{0}\right)+i_{3}\left(x_{1} w_{3}+(2 m-1) x_{3} w_{1}\right)\right)
$$

where

$$
w=w_{0}+w_{1} i_{1}+w_{2} i_{2}+w_{3} i_{3}
$$

is the corresponding pseudoanalytic function of the first kind. Using (15) with $w$ in the form (13) we finally get a representation of the solutions of the differential equation (14) using differential operators.

\section{References}

1. L. Bers, Theory of Pseudo-Analytic Functions, New York University, 1953,

2. K. Gürlebeck, W. Sprössig, Quaternionic and Clifford Calculus for Physicists and Engineers, John Wiley, Chichester, 1997,

3. V.V. Kravchenko, On the reduction of the multidimensional stationary Schrödinger equation to a first-order equation and its relation to the pseudoanalytic function theory, J. Phys. A 38 (2005), 851-868,

4. V.V. Kravchenko, H.R. Malonek, G. Santana, Biquaternionic integral representations for massive Dirac spinors in a magnetic field and generalized biquaternionic differentiability, Math. Methods Appl. Sci. 19 (1996), 1415-1431,

5. H.R. Malonek, Remarks on a spatial version of the pseudoanalytic functions in the sense of Bers, W. Sprössig, K Gürlebeck (eds.), Analytical and Numerical Methods in Quaternionic and Clifford Analysis, 113-119, Proc. Symposium, Seiffen, 1996,

6. H.R. Malonek, Generalizing the $(F, G)$-derivative in the sense of Bers, in: V. Dietrich, K. Habetha, G. Jank (eds.), Clifford Algebras and their Application in Mathematical Physics, 247-257, Kluwer, Dordrecht, 1998,

7. I.N. Vekua, Verallgemeinerte analytische Funktionen, Akademie Verlag, Berlin, 1963. 